\title{
Osteotomien - Distaler Unterschenkel und Sprunggelenk
}

\author{
Hans Zwipp, René Grass, Stefan Rammelt
}

\section{Zusammenfassung}

Nach OSG-gelenknahen Unterschenkelfrakturen (43 A1-A3 nach AO) sowie Pilon- und OSG-Frakturen (43 B1-B3, 43 C1-C3, 44 A1-C3) kann es sowohl nach konservativer als auch nach operativer Behandlung zu Fehlstellungen supramalleolar, malleolar, intraartikulär und fortgeleitet auch inframalleolar kommen. Häufig gibt es Kombinationen der 4 Grundtypen, welche Fehlstellungen im Sinne des Varus, des Valgus, der Ante- oder Retrokurvation und/ oder die Malrotation betreffen. Im Bereich des Außenknöchels kommt in Bezug auf Osteotomien insbesondere der posttraumatischen Fibulaverkürzung eine bedeutende Rolle zu. Präoperativ sind Belastungsaufnahmen des oberen Sprunggelenks a.-p. beidseits mit $20^{\circ}$ Innenrotationsstellung der Füße, seitliche Fußaufnahmen mit oberem Sprunggelenk beidseits sowie Rückfußaufnahmen nach Saltzman unabdingbar. Nach der Literatur gelten als Indikationen zur supramalleolaren Korrekturosteotomie Achsabweichungen $\geq 5^{\circ}$ Varus, $\geq 10^{\circ}$ Valgus, $>15$ bis $20^{\circ}$ Ante-/Retrokurvation sowie eine Mal- rotation $\geq 10$ bis $20^{\circ}$. Im eigenen Vorgehen werden Malrotationen der Fibula $\geq 5^{\circ}$ korrigiert, Fibulaverkürzungen von mehr als $2,5 \mathrm{~mm}$, wenn dadurch eine Gabelinstabilität induziert wird sowie Gelenkstufen $\geq 2 \mathrm{~mm}$. Zur Osteosynthese von subtraktiven und additiven Osteotomien reichen 3,5 winkelstabile und auch nicht winkelstabile Plattenosteosynthesen im Sprunggelenksbereich in der Regel aus. Bei komplexen Fehlstellungen und bei „dome shape“-Osteotomien kann ein Ilizarovoder Hexapod-Fixateur zur Anwendung kommen.

\section{Osteotomies of the Distal Lower Leg and Ankle}

Distal fractures of the lower leg (43 A1A3), fractures of the pilon (43 B1-B3, $43 \mathrm{C} 1-\mathrm{C} 3)$ and fractures of the ankle (44 A1-C3) can result, after conservative or operative treatment, in malunions at the supramalleolar, malleolar or intraarticular level. Malunions may concern varus, valgus, ante- or retrocurvation deformity and/or a malrotation. Preoperatively, weight-bearing X-rays of the ankle joint in a.p. with mortise view projection, both lateral feet with ankle are highly recommended as well as the hindfoot view according to Saltzman which is absolutely needed for preoperative planning. With regard to malunions of the lateral malleolus, most often a significant shortening requires an oblique osteotomy or interposition of a cortico-cancellous bone graft for lengthening. According to the literature a supramalleolar osteotomy is recommended in cases $\geq 5^{\circ}$ varus, $\geq 10^{\circ}$ valgus, $\geq 15$ to $20^{\circ}$ ante-/retrocurvation or a malrotation of $\geq 10$ to $20^{\circ}$. According to our own decision-making algorithm, a malrotation of the fibular is corrected in cases $\geq 5^{\circ}$, or shortening of $\geq 2.5 \mathrm{~mm}$ if lateral shifting of the talus with widening of the medial clear space is given. An intraarticular step of $\geq 2 \mathrm{~mm}$ needs an osteotomy with correction of the joint congruency. For stabilising closing or opening wedge osteotomies, 3.5 locking or non-locking plates are recommended. In complex deformities and with the necessity of a dome-shaped osteotomy, the correction may be done by means of an Ilizarov or a Hexapod fixator.

\section{Einleitung}

Fehlstellungen des distalen Unterschenkels und/oder des Sprunggelenks gehen in der Regel auf fehlverheilte konservativ oder operativ behandelte gelenknahe Unterschenkelbrüche, Pilon- und/oder Sprunggelenksfrakturen zurück. Korrekt behandelte OSG-Frakturen im Kindesalter können bei Crush-Verletzung der distalen Epiphyse zum vorzeitigen Verschluss und konsekutivem Fehlwachs-

OP-JOURNAL 2011; 27: 260-267

(c) Georg Thieme Verlag KG Stuttgart · New York DOI http://dx.doi.org/10.1055/s-0031-1280429 tum tibial oder fibular führen. Idiopathische Fehlstellungen der OSG-Achse und/ oder des Rückfußes sind nicht selten bei chronischer fibularer Bandinstabilität oder beim fehlangelegten Kugeltalus beobachtbar. Relevante supramalleolare Varus-, Valgus-, Ante- oder Retrokurvationsfehlstellungen von distaler Tibia und/oder Fibula sowie Malrotationen oder Verkürzungen der Fibula, seltener des Malleolus medialis, erfordern ebenso wie fixierte Gelenkstufen ein klares Behandlungskonzept, was im Folgenden beschrieben werden soll.

\section{Diagnostik}

Liegt eine isolierte posttraumatische Fehlstellung im unteren Drittel des Unterschenkels vor, sind in der Regel keine Achsabweichungen in Höhe des Hüftoder Kniegelenks zu erwarten, sodass in diesen Fällen auf Ganzbeinaufnahmen im Stehen verzichtet werden kann [3].

Bei fehlverheilten Pilon- und OSG-Frakturen sind Belastungsaufnahmen des oberen Sprunggelenks beidseits a.-p. im Stehen mit $20^{\circ}$ Innenrotation, exakt seitliche Projektionen des Fußes mit oberem Sprunggelenk beidseits unter Körperlast als auch sogenannte Saltz- 


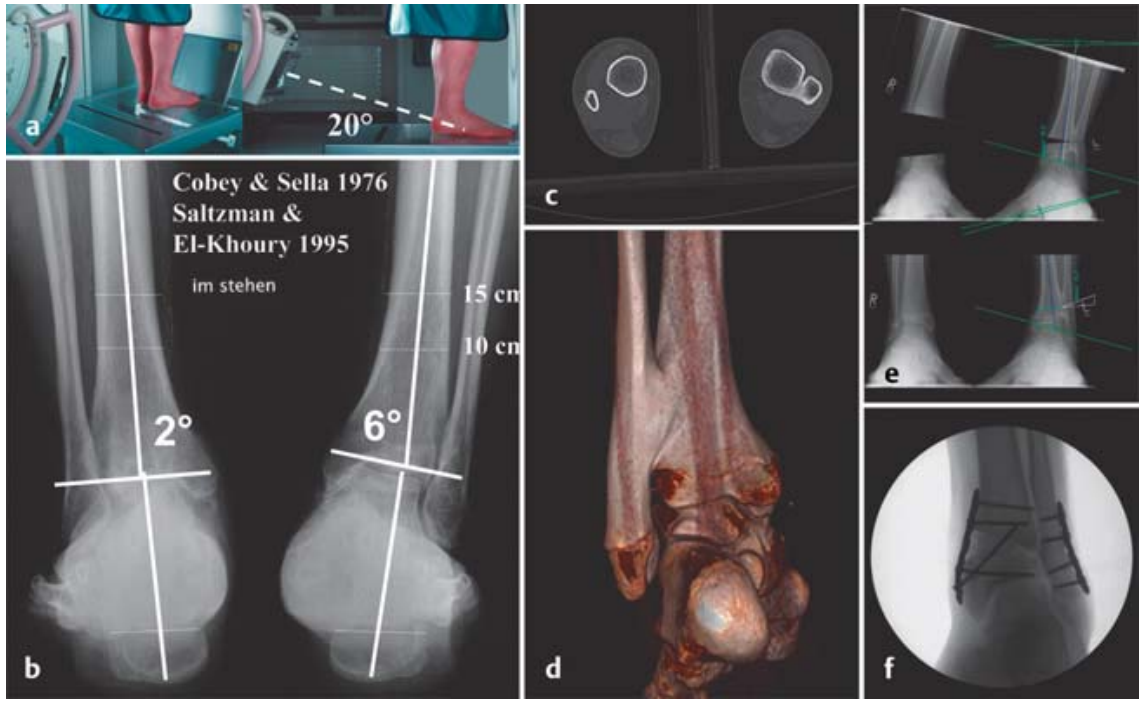

Abb. $\mathbf{1}$ a bis $\mathbf{f}$ Diagnostik und Planung der supramalleolaren Osteotomie. a, b Die Rückfußaufnahmen nach Cobey und Sella [1], modifiziert nach Saltzman und El-Khoury [7], werden im Stehen durchgeführt. Die Röntgenplatte ist ebenso wie der Röntgenstrahl um $20^{\circ}$ geneigt. Die mittlere Tibiaachse wird 10 und $15 \mathrm{~cm}$ oberhalb der OSG-Ebene zentriert eingezeichnet, die in Höhe der Tibiabasislinie auf die gemittelte Rückfußachse des Fersenbeins trifft. c, d Bei supramalleolaren Fehlstellungen ist vor der Korrektur eine Feinschicht-CT-Untersuchung durchzuführen, wenn wie hier gezeigt eine vollständige Synostose von einer partiellen unterschieden werden muss. Die 3-D-Darstellung macht den notwendigen Zugangsweg von dorsal besonders verständlich. e Die präoperative Planung für die supramalleolare Osteotomie kann anhand der OSG-Belastungsaufnahmen im Stehen mit digitalem Planungsprogramm bearbeitet werden. $\mathbf{f}$ Die intraoperative Durchleuchtung zeigt nach Auflösung der Synostose die achsengerechte Korrektur von Tibia und Fibula.

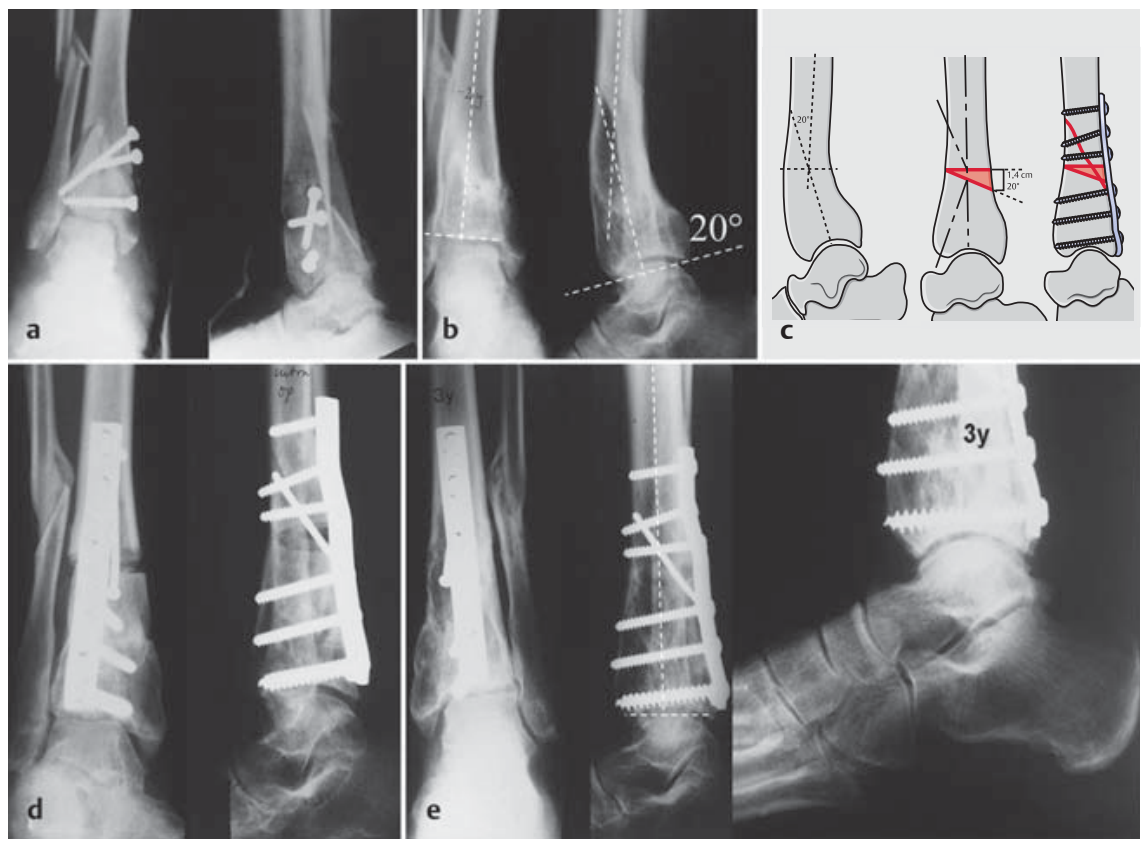

Abb. 2a bis e Beispiel einer fehlverheilten Pilon-tibiale-Fraktur mit supramalleolarer Fehlstellung 2 Jahre nach Erstversorgung mit $20^{\circ}$ Antekurvationsfehlstellung. (a) Konsekutive Ausheilung in $6^{\circ}$ Valgus und $20^{\circ}$ Antekurvationsfehlstellung mit klinisch starker Beschwerdesymptomatik im ventralen oberen Sprunggelenk bei Impingement, insbesondere beim Treppaufsteigen (b). Die additive Osteotomie im Zentrum der Fehlstellung wird über einen dorsolateralen Zugang mit Interposition eines an der Basis $14 \mathrm{~mm}$ hohen kortikospongiösen Spanes unter Stabilisierung mit einer breiten DC-Platte geplant (c), um die $20^{\circ}$-Fehlstellung vollständig auszugleichen (c). Das postoperative Röntgenbild (d) zeigt die achsengerechte Korrektur einschließlich der Schrägosteotomie an der Fibula. Beim 3-Jahres-Follow-up (e) ist die Patientin bei korrigierter Achse völlig beschwerdefrei, wenngleich der physiologische Slope nicht vollends erreicht wurde.
man-Aufnahmen (Abb. $\mathbf{1}$ a, b) für die Erkennung der Pathologie und die präoperative OP-Planung unabdingbar.

Liegen Hinweise für eine tibiofibulare Synostose oder zusätzliche Gelenkstufe vor, ist zusätzlich eine Feinschicht-CTUntersuchung in 2 Ebenen, gegebenenfalls mit 3-D-Rekonstruktion (Abb. 1 c, d), notwendig.

Zu beachten gilt, dass die Tibiabasislinie zur Tibiaschaftachse physiologischerweise lateral einen Winkel von $89 \pm 3^{\circ}$ (LDTA $=$ lateral distal tibial angle) hat, die seitliche Tibiagelenkflächenlinie zur seitlichen Tibiaschaftachse einen ventralen Winkel (aADTA = anatomic anterior distal tibial angle) von nur $80^{\circ}$ hat, d.h. der sog. „Slope“ des oberen Sprunggelenks von dorsal nach ventral beträgt $10^{\circ}[6]$.

Bei OSG-a.-p.-Aufnahmen im Stehen muss eine mögliche Taluskippung (talar tilt) nach außen oder innen mitbetrachtet werden. In Bezug auf die Fibulalänge gilt zu beachten, dass der OSG-Achsenwinkel von „tip to tip“ im „mortise view“ $82 \pm 3,6^{\circ}$ beträgt [6].

Bei chronischer Syndesmoseninsuffizienz sind nach eigener Erfahrung bei a.-p. Belastungsaufnahmen nicht nur die Fibulalänge im Seitenvergleich, sondern vor allem der "espace clair“ $(\leq 5 \mathrm{~mm})$, der "medial clear space“ (>2,5 mm) und das sog. „overlapping“ der Fibula über die Tibia (> $5 \mathrm{~mm}$ ) zu beachten.

\section{Indikation}

Als allgemeine Empfehlung für eine supramalleolare Korrekturosteotomie werden Achsabweichungen von $>5^{\circ}$ Varus, $>10^{\circ}$ Valgus, $>15-20^{\circ}$ Ante-/Retrokurvation und eine Malrotation $>10-20^{\circ}$ angegeben, wobei die Innenrotation von mehr als $10^{\circ}$ nicht toleriert werden soll $[2-5,10-13]$.

Im eigenen Vorgehen wird bei fehlverheilten Knochenbrüchen eine Malrotation der Fibula von $\geq 5^{\circ}$ korrigiert, eine Ante-/Retrokurvation $>10^{\circ}$, eine Verkürzung der Fibula von mehr als $2 \mathrm{~mm}$, wenn dadurch der "medial clear space“ $>2,5 \mathrm{~mm}$ und der Espace Clair $>5 \mathrm{~mm}$ erweitert wird. Intraartikuläre Stufen $\geq 2 \mathrm{~mm}$ sowie eine „Slope“-Abweichung um mehr als $10^{\circ}$ werden im eigenen Vorgehen ebenfalls korrigiert. Fehlstellungen der Fibula in der koronaren CT-Ebene in Bezug auf die Incisura fibularis ti- 
biae mit einer Abweichung von mehr als $2 \mathrm{~mm}$ nach ventral, dorsal oder lateral werden ebenfalls als pathologisch betrachtet und sind im Rahmen der Gesamtdiagnostik der chronischen Syndesmoseninsuffizienz als gesonderte Entität zu betrachten.

\section{Präoperative Planung und Operation}

Die operative Planung, ob additiv oder subtraktiv korrigiert werden soll, hängt oftmals von den Weichteilen, der Komplexität oder der Gesamtsituation des Patienten ab.

Individuelle OP-Skizzen beinhalten den Ist- und Soll-Zustand, die vom Operateur selbst gefertigt werden sollen.

Digitale Planungsprogramme erleichtern das Zeichnen (Abb.1e). Als operative Therapie kommen Opening- und Closing-Wedge-Osteotomien der Tibia, Schräg-, Treppen- und Querosteotomien der Fibula ohne und mit autologer kortikospongiöser Beckenkammspaninterposition in Betracht. Die osteosynthetische Stabilisierung erfolgt in der Regel mit 3,5 mm LCDCP, Reko- oder winkelstabilen Platten. Gelegentlich kann auch bei einer „dome shape“-Osteotomie mit Distraktions-, Rotationsosteotomie ein Ilizarov- oder Hexapod-Fixateur-System zur Anwendung kommen. Bei Fehlstellungen mit zusätzlicher Synostose zwischen Fibula und Tibia wird diese im eigenen Vorgehen grundsätzlich reseziert, um diese potenzielle präarthrotische Deformität durch Wiederherstellung des freien Fibulaspiels zu beseitigen (Abb. 1 c-f).

Im Folgenden sollen Beispiele von supramalleolaren, malleolaren und/oder Gelenkfehlstellungen sowie kombinierte Achsabweichungen der OSG-Ebene, des Talus und/oder des gesamten Rückfußes beschrieben werden.

\section{Supramalleolare Anteversionsfehlstellung}

Tarr und Mitarbeiter [9] konnten bereits 1985 experimentell zeigen, dass die tibiotalare Gelenkfläche in Neutralstellung bei einer Antekurvation von nur $10^{\circ}$ oder einer Rekurvationsfehlstellung von $15^{\circ}$ bereits um mehr als 30\% reduziert wird. Die Abb. 2 zeigt einen Fall einer 46-jährigen Patientin 2 Jahre nach minimalistisch versorgter Schraubenosteosynthese einer Pilon-tibiale-C1-Fraktur.
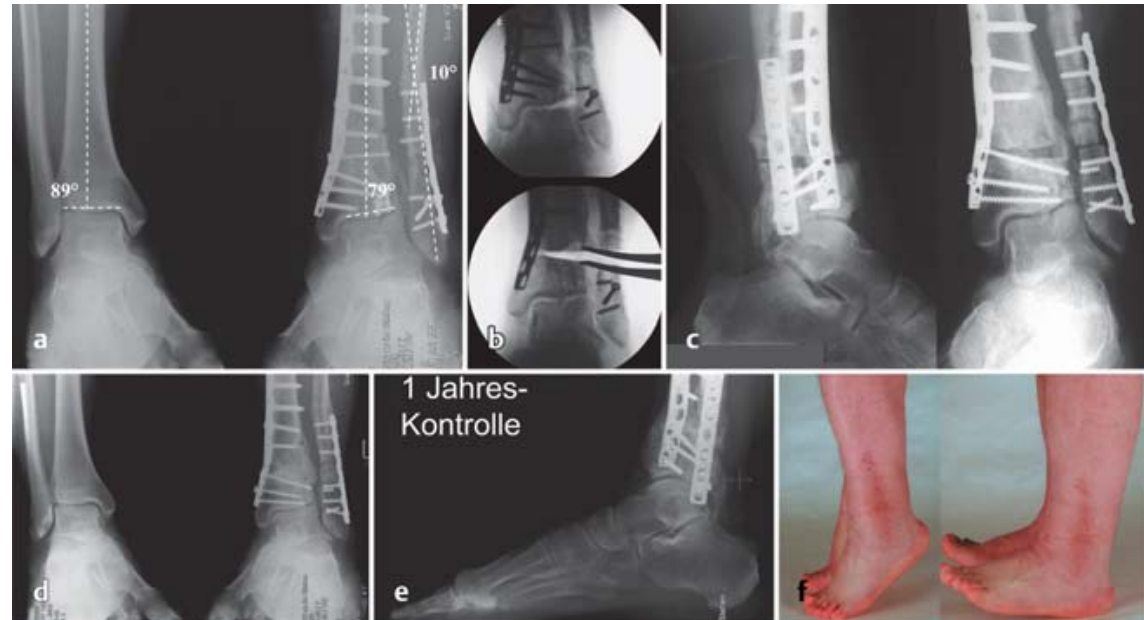

Abb. 3 a bis $\mathbf{f}$ Supramalleolare Valgusfehlstellung 6 Monate nach operativer Versorgung einer Pilon-tibiale-C2-Fraktur aufgrund einer Osteoporose der lateralen Tibiametaphyse (a). Entsprechend der präoperativen Planung wird transfibulär von lateral $3 \mathrm{~cm}$ oberhalb der Gelenkebene nach Entfernen der distalen, durch die mediale LCDCP eingebrachten Schrauben die OpeningWedge-Osteotomie von $10^{\circ}$ mit Interposition zweier autologer kortikospongiöser Blöcke in Sandwich-Technik vorgenommen (b), dies mit zusätzlich achskorrigierender Re-Osteosynthese der Fibula (c). Die 1-Jahres-Kontrolluntersuchung (d-f) zeigt bis auf einen minimalen Restvalgus eine gute Verheilung mit schmerzfreier, guter Funktion im oberen Sprunggelenk.
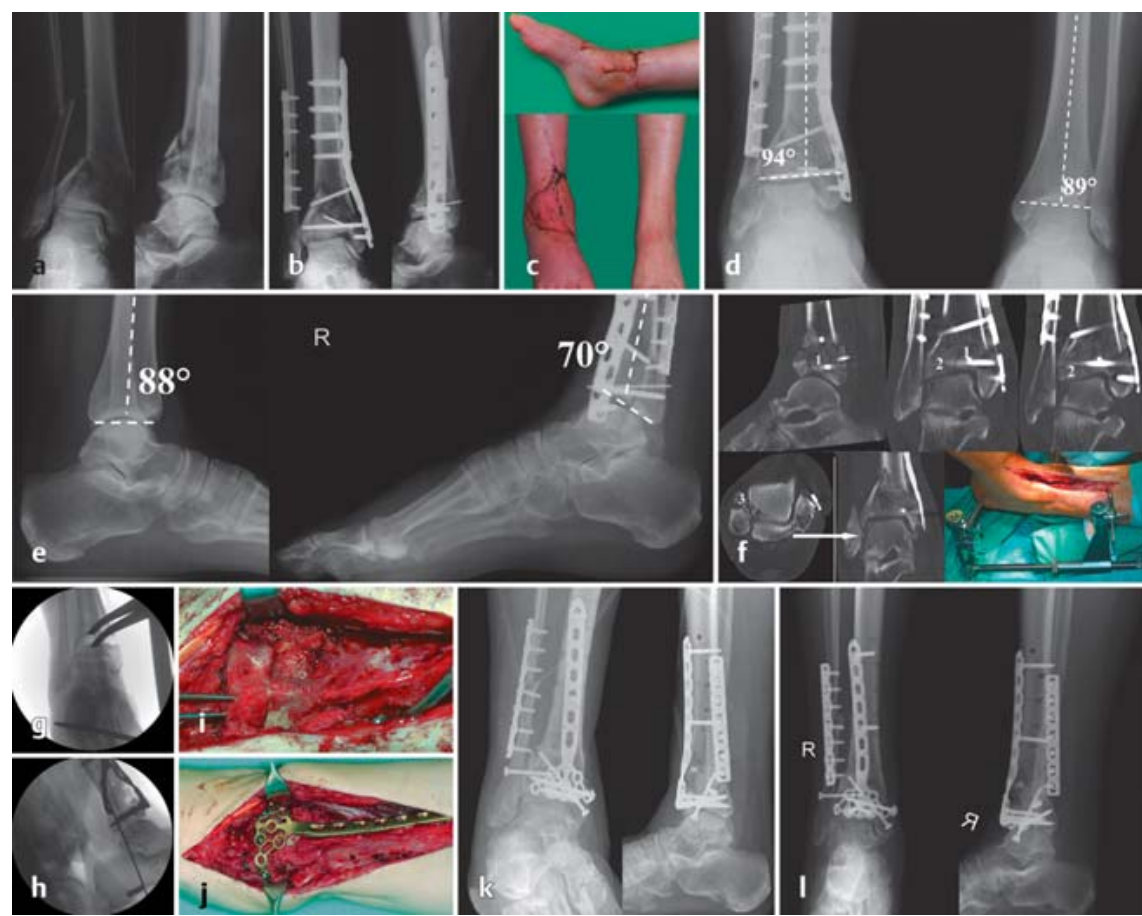

Abb. 4 a bis I (a) Beispiel einer Varusfehlstellung der OSG-Ebene (LDTA), kombiniert mit pathologischem ADTA bzw. OSG-Slope und Gelenkstufe 3 Monate nach Pilon-C2-Fraktur. Bereits die postoperative Aufnahme zeigt die Varusfehlstellung der Gelenkebene sowie den pathologischen Slope (b), die sich 3 Monate später nach 5 vorausgegangenen Operationen einschließlich Schwenklappen (e) konsolidierten. Im Rahmen einer Heilverfahrenskontrolle zeigen die Belastungsaufnahmen im Seitenvergleich eine Varusfehlstellung von $5^{\circ}$ der Gelenkebene $\left(94^{\circ}\right.$ rechts versus $89^{\circ}$ links) sowie einen pathologischen Slope von $18^{\circ}$ ( $88^{\circ}$ links versus $70^{\circ}$ rechts, $\mathbf{d}, \mathbf{e}$ ). Wegen der vermuteten intraartikulären Gelenkstufe wird zur Operationsplanung zusätzlich noch eine Feinschicht-CT-Untersuchung notwendig (f), welche das nicht reponierte zentrale „die punch“-Fragment, die Varusfehlstellung des Tubercule-de-Chaput-Fragments, ein interponiertes Fragment in der Incisura fibularis tibiae sowie einen kleinen freien Gelenkkörper im fibulotalaren Gelenkspalt zeigt (f, Ziffern 1-3, weißer Pfeil). Zur Korrektur der Varus- und Slope-Fehlstellung ist der Einsatz eines Femurdistraktors (f) unerlässlich. Durch das ventrale Öffnen mittels Osteotomie $2 \mathrm{~cm}$ oberhalb der Gelenkebene kann mit Interposition von 2 kortikospongiösen Spänen in Sandwich-Technik und durch ventrale Abstützung mittels winkelstabiler Pilon-tibiale-Platte $(\mathbf{g}-\mathbf{j})$ die Achse korrigiert werden. Das postoperative Röntgenkontrollbild nach zusätzlicher Korrektur der Fibula und Ausräumen der Inzisur mit Entfernung des freien Gelenkkörpers (k) zeigt die Wiederherstellung von LDTA, ADTA und des Gelenks mit guter Verheilung bereits nach 2 Monaten (I). 


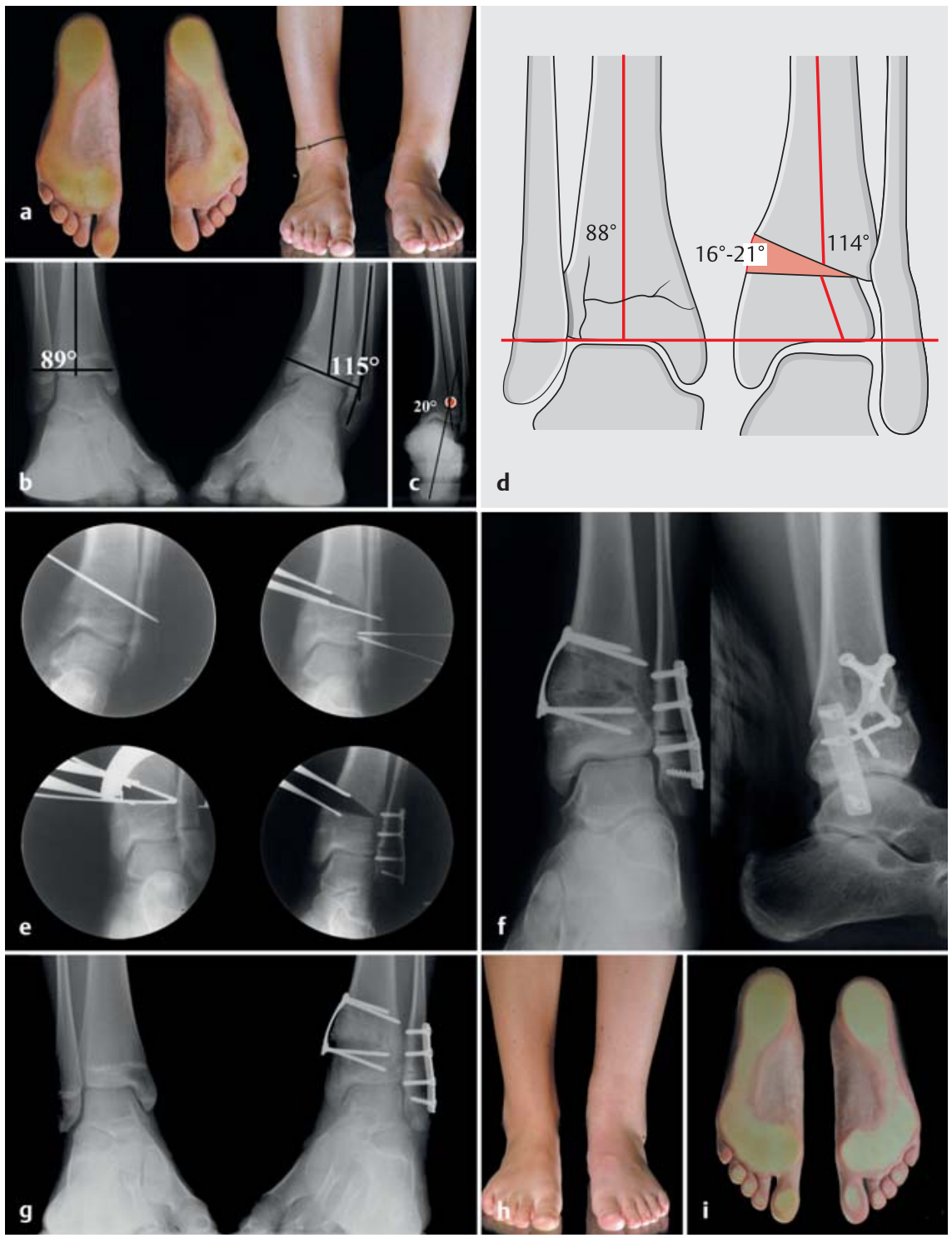

Abb. 5a bis i Fehlstellung der OSG-Ebene und des Außenknöchels mit Gesamtfehlstellung des Rückfußes von $20^{\circ}$ nach posttraumatischer, vorzeitiger tibialer Epiphysiodese. Die Abb. $\mathbf{5}$ a-d zeigen den Zustand bei einem 15-jährigen Mädchen 5 Jahre nach Salter-Harris-5-Fraktur mit einem LDTA von $115^{\circ}$ links gegenüber $89^{\circ}$ rechts und einer Gesamtachsenfehlstellung des Rückfußes gegenüber der Tibiaschaftachse im „center of rotation and angulation“ (CORA) von $20^{\circ}$ (b,c). Entsprechend der präoperativen Planung (d) sind eine Opening-Wedge-Osteotomie von $20^{\circ}$ sowie eine Closing-Wedge-Osteotomie der Fibula vorgesehen. Intraoperativ wird nach paralleler Osteotomie $3 \mathrm{~cm}$ oberhalb der Gelenkebene im metaphysären Bereich der Osteotomiespalt mit dem Arthrodesenspreizer soweit aufgeweitet, dass die mit $20^{\circ}$ Keilform entnommenen bikortikalen Beckenkammspäne in Sandwich-Technik eingebracht werden können. Nach analoger Closing-Wedge-Osteotomie der Fibula wird die Tibia medial mit winkelstabiler X-Platte und die Fibula lateral mit einfacher 4-Loch-Drittelrohrplatte stabilisiert (e,f). Die 4-Monats-Kontrolle (g-i) zeigt die achsengerechte Wiederherstellung von Tibia und Fibula, was insbesondere auch durch die klinische Spiegeltischaufnahme (i) im Vergleich zur präoperativen Spiegeltischaufnahme (a) zum Ausdruck kommt.

\section{Supramalleolare Valgusfehlstellung}

Nach Pilon-tibiale-Frakturen kommt es nicht selten bei sekundärer Sinterung des lateralen Gelenkblocks zur Valgusfehlstellung der Talusbasislinie, nicht selten kombiniert mit einer Valgusabknickung des Wadenbeins wie im vorliegenden Fall (Abb. 3).

Varusfehlstellung der OSG-Ebene (LTDA), kombiniert mit pathologischem (ADTA) OSG-Slope und Gelenkstufe

Nach Pilon-tibiale-Frakturen kommt es nicht selten primär oder sekundär zur Fehlstellung der Gelenkebene oder des Gelenks selbst, sodass Frühkorrekturen angezeigt sind. Das Fallbeispiel (Abb.4) zeigt den Zustand einer 52-jährigen $\mathrm{Pa}$ tientin 3 Monate nach einer Pilon-C2Fraktur, zweitgradig offen, im Rahmen eines Sturzes von einer Kletterwand bei physiotherapeutischer Behandlung wegen Rückenschmerzen (Abb.4a).

Fehlstellung der OSG-Ebene nach posttraumatischer Epiphysiodese

Bei einer Crush-Verletzung der distalen Epiphyse der Tibia bei kindlicher OSGFraktur kann wie im vorliegenden Fall (Abb.5) trotz korrekter primärer operativer Versorgung die vorzeitige Epiphysiodese einsetzen, die zur Fehlstellung der OSG-Ebene und zur konsekutiven Überlänge sowie Varusfehlstellung der Fibula führt.

\section{Fibulaverkürzung nach Maisonneuve-/} PE-3-/-4-/Weber-C-Fraktur

Eine der am häufigsten notwendigen supramalleolaren Osteotomien ist die Schrägosteotomie der Fibula zur Verlängerung und/oder Achsenkorrektur derselben nach unzureichender operativer/ konservativer Versorgung. Im vorliegenden Fallbeispiel einer 56-jährigen Patientin zeigt sich bereits im intraoperativen Röntgenbild des oberen Sprunggelenks a.-p. links nach Einzeichnen des sog. Weberkreises die Verkürzung der Fibula nach Fixation derselben mit nur 1 Syndesmosenstellschraube (Abb. 6).

\section{Rotationsfehlstellung der Fibula}

Auch bei vermeintlich harmlosen Weber-B-Frakturen kann durch Fixation des Außenknöchels in Malrotation ein relevantes Problem wie im Fallbeispiel (Abb. 7) gesehen werden. 

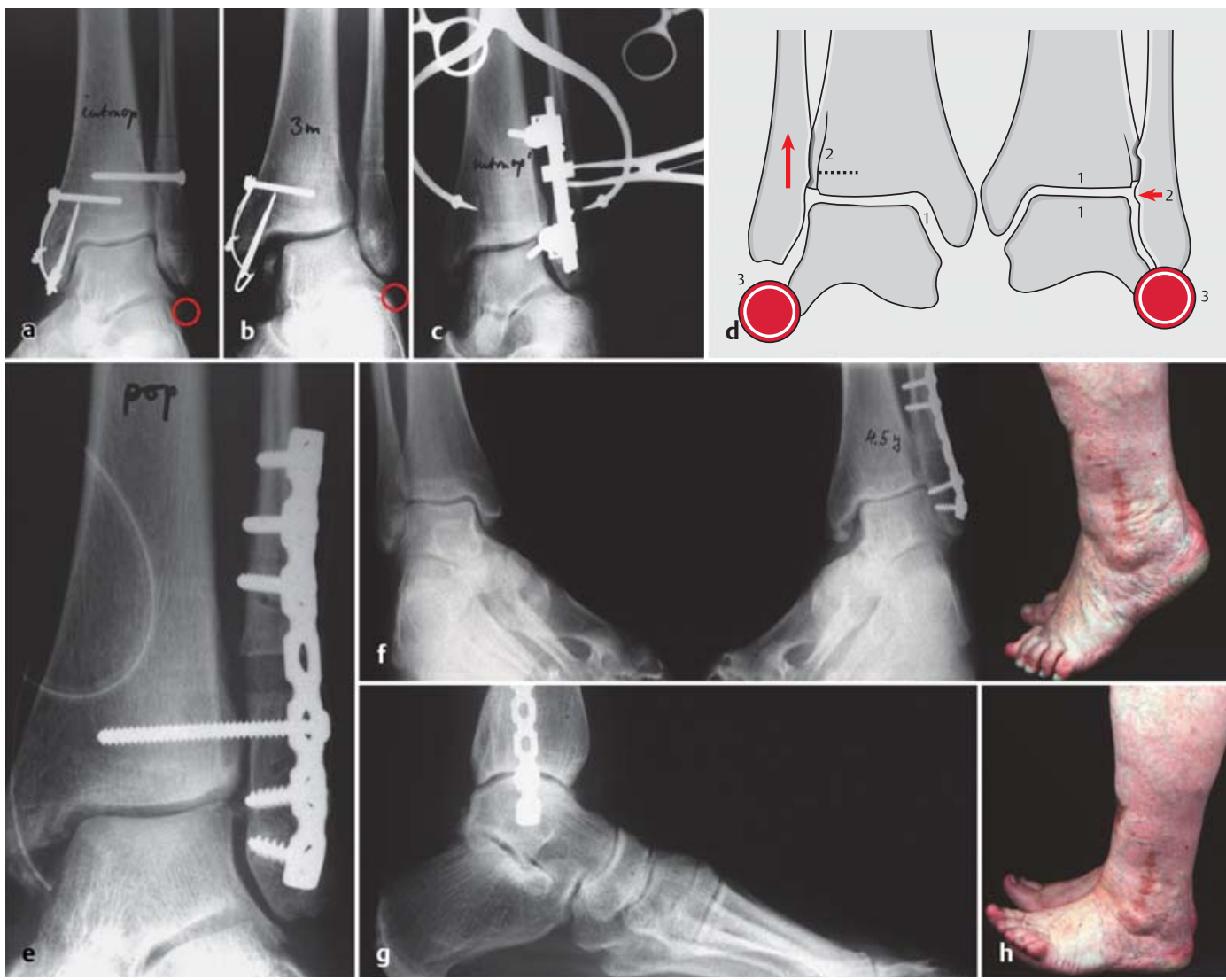

Abb. $\mathbf{6 a}$ bis $\mathbf{h}$ Klassische Fibulaverkürzung nach Maisonneuve-Fraktur bereits intraoperativ (a) erkennbar. Bereits 3 Monate später führt die fehlverheilte und verkürzte Fibula zur Gabelinstabilität mit massivem Shift des Rückfußes nach lateral unter Aufweitung des „espace clair“ und Erweiterung des „medial clear space“ von über $1 \mathrm{~cm}(\mathbf{b})$. Unter Kontrolle des einzuzeichnenden Weber-Balles zeigt sich intraoperativ (c-e), dass eine Verlängerung der Fibula durch Distalisierung derselben mittels Verschiebung der distal an der LCDCP fixierten Fibula und Spreizen des mittelgroßen Arthrodesenspreizers zwischen proximalem Plattenrand und der proximal davon eingebrachten freien Schraube (c) mit Interposition eines kortikospongiösen Spanes von 9 mm Höhe notwendig ist. Durch erneutes Einpassen der Fibula in die Inzisur nach Revision derselben und Setzen einer Syndesmosenstellschraube nach Längenwiederherstellung der Fibula und Einpressen derselben in die Inzisur mit der Spitz-Spitz-Zange gelingt der stabile Gabelschluss. Das 41/2-Jahres-Kontrollergebnis (f) lässt radiologisch keine signifikante Arthrose erkennen, der „espace clair“ ist minimal erweitert, die Funktion für Streckung und Beugung ist gut, die Patientin ist schmerzfrei.

\section{Relevante Fehlverheilungen des Volkmann-Fragments}

Kommt es durch unzureichende anatomische Reposition eines großes Volkmann-Fragments zur Stufenbildung, die einen Shift des gesamten Fußes mit Talus nach dorsal bedingt, ist eine Osteotomie und Korrektur des Volkmann-Fragments zur Vermeidung einer frühen posttraumatischen Arthrose indiziert. Im folgenden Fallbeispiel (Abb. 8) soll der Fall eines 37-jährigen Patienten gezeigt werden, der 1 Jahr zuvor wegen einer trimalleo- laren OSG-Fraktur (Weber C) operativ versorgt worden war.

OSG- und Rückfußvarus bei chronischer anterolateraler Rotationsinstabilität des oberen Sprunggelenks

Bei ossärem Bandausriss im Kindesund Jugendalter und konsekutiver chronischer Instabilität kann es wie im folgenden Fallbeispiel (Abb.9) zur Abweichung der OSG-Basislinie mit begleitendem Rückfußvarus kommen.
Chronische anterolaterale Rotationsinstabilität (ALRI) mit fixierter Varuskippung des Talus

Die Korrektur eines über Jahre hinweg pathologisch in der OSG-Gabel fixierten Talus in Varusfehlstellung führt zur Dekompensation des gesamten Rückfußes in eine Varusfehlstellung bis $30^{\circ}$ (Abb. 10) und konsekutiver Kontraktur des Deltoids sowie progredienter Arthrose des medialen Kompartiments. 


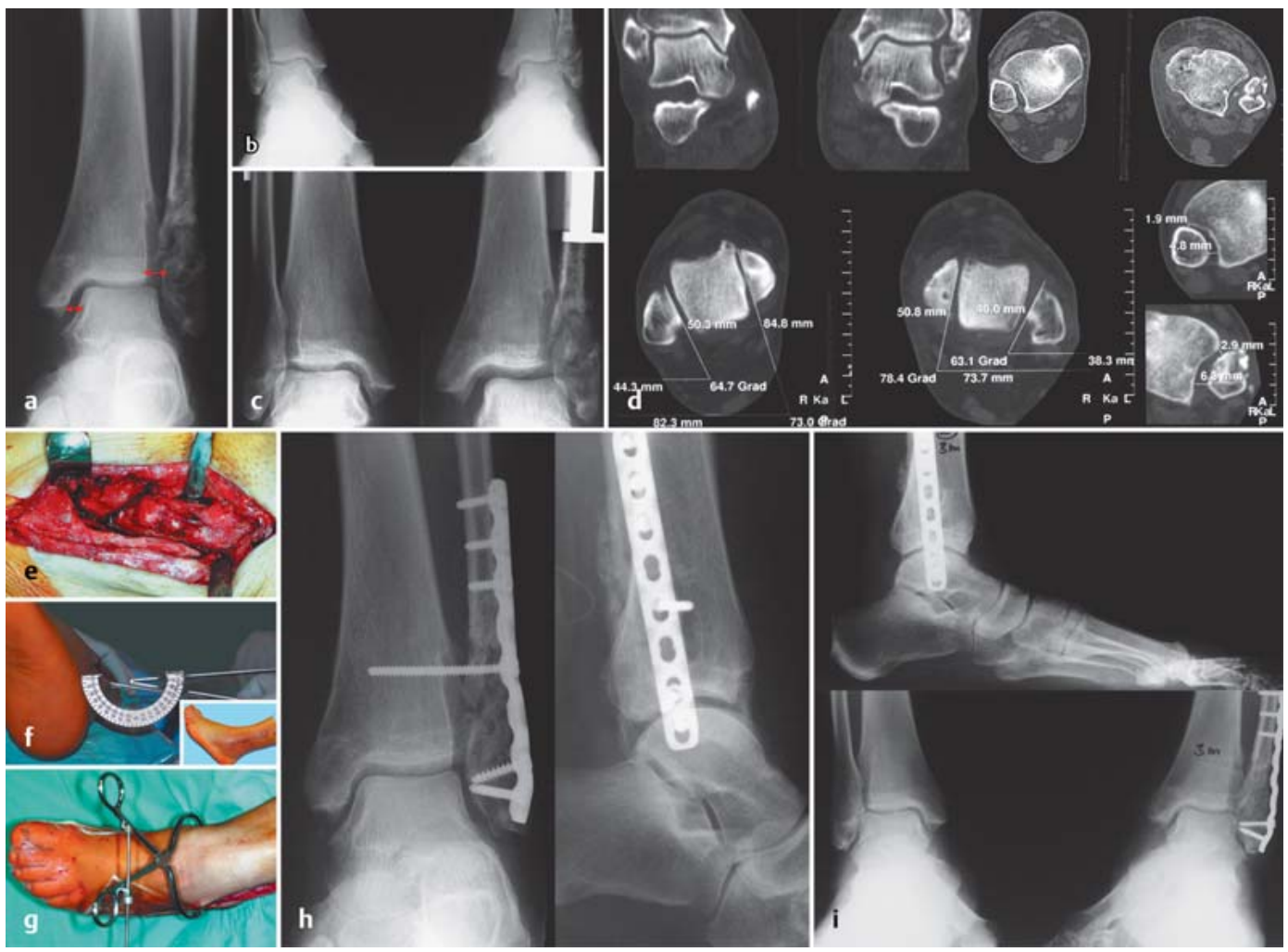

Abb. 7 a bis i Sechs Monate nach Primärversorgung einer monomalleolaren Weber-B-Fraktur mit zwischenzeitlich entfernter Platte (wegen Wundheilungsstörungen) besteht eine „malunion“ und „nonunion“ des Außenknöchels mit geringer Verkürzung, Shift des Talus nach außen und gering erweitertem „espace clair“ und erweiterten „medial clear space“ (a, rote Pfeile). Die Belastungsaufnahmen des oberen Sprunggelenks a.-p. im Stehen beidseits (b) zeigen bei der 45-jährigen Patientin weniger die Pathologie als die Syndesmosenstressaufnahmen mit deutlicher Erweiterung des „medial clear space“ (c). Die präoperative CT-Planung im Seitenvergleich (d) zeigt vor allem die $13^{\circ}$-Außenrotationsfehlstellung der Fibula und den Versatz der Fibula nach außen, nach lateral und ventral unter erheblicher Entfernung von der Incisura fibularis tibiae. Intraoperativ wird nach Lösen der Fehlverheilung (e) und initialem Setzen von 2 K-Drähten die 13-Außenrotationsfehlstellung markiert (f). Die Rotationskorrektur des distalen Fibulaanteils erfolgt durch Drehen, bis beide Drähte parallel stehen, um dann mit autologer Spongiosa und einer 8-Loch-LCDCP + Stellschraube die Korrektur zu sichern (g, h). Das Halbjahreskontrollröntgenbild zeigt die gute Ausheilung (i).

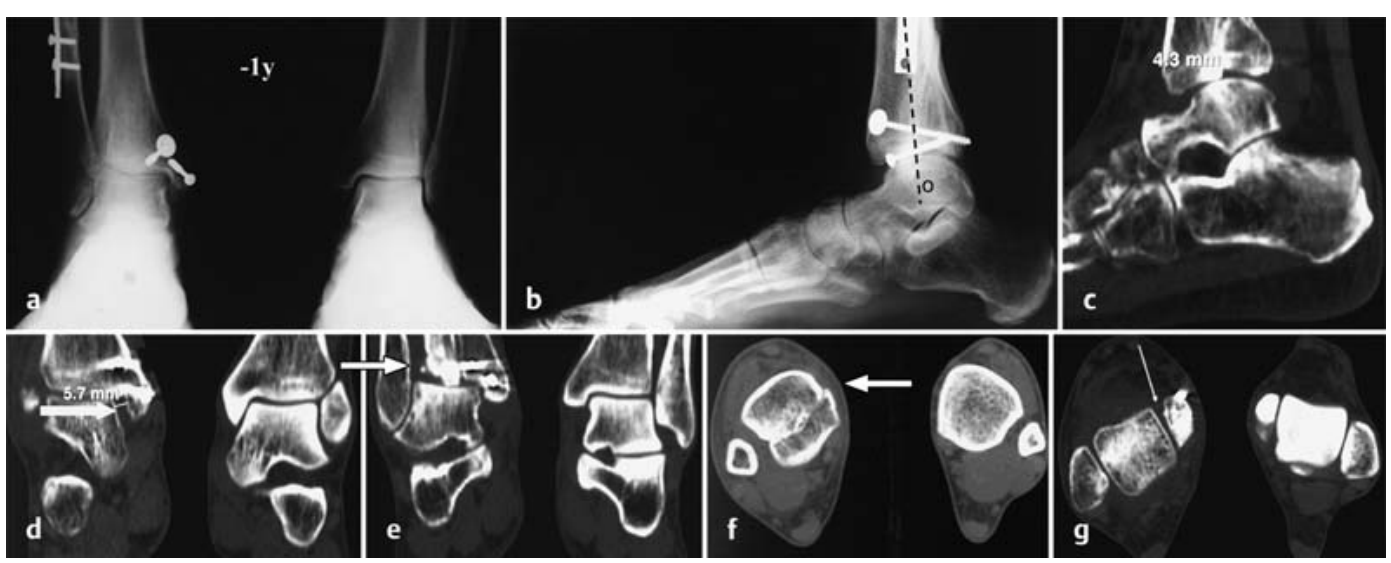

Abb. $\mathbf{8}$ a bis $\mathbf{g}$ Relevante Fehlverheilung eines großen Volkmann-Fragments 1 Jahr nach trimalleolarer, operativ versorgter Weber-C-Fraktur. Die Belastungsaufnahmen zeigen bei noch liegender Drittelrohrplatte und verschraubtem Innenknöchel die Subluxation des gesamten Fußes nach dorsal (a,b), was durch das fehlende Widerlager bei höhergetretenem Volkmann-Dreieck, vor allem in der CT-Aufnahme (c), in der sagittalen Rekonstruktion gut zum Ausdruck kommt. Die frontale Rekonstruktion zeigt die Erweiterung des „medial clear space“ (d), die Erweiterung des "espace clair“ (e) und den Versatz der hinteren Hälfte des Tibiaplafonds nach medial (f) sowie die Außenrotationsfehlstellung des Innenknöchels um $10^{\circ}(\mathbf{g})$. 

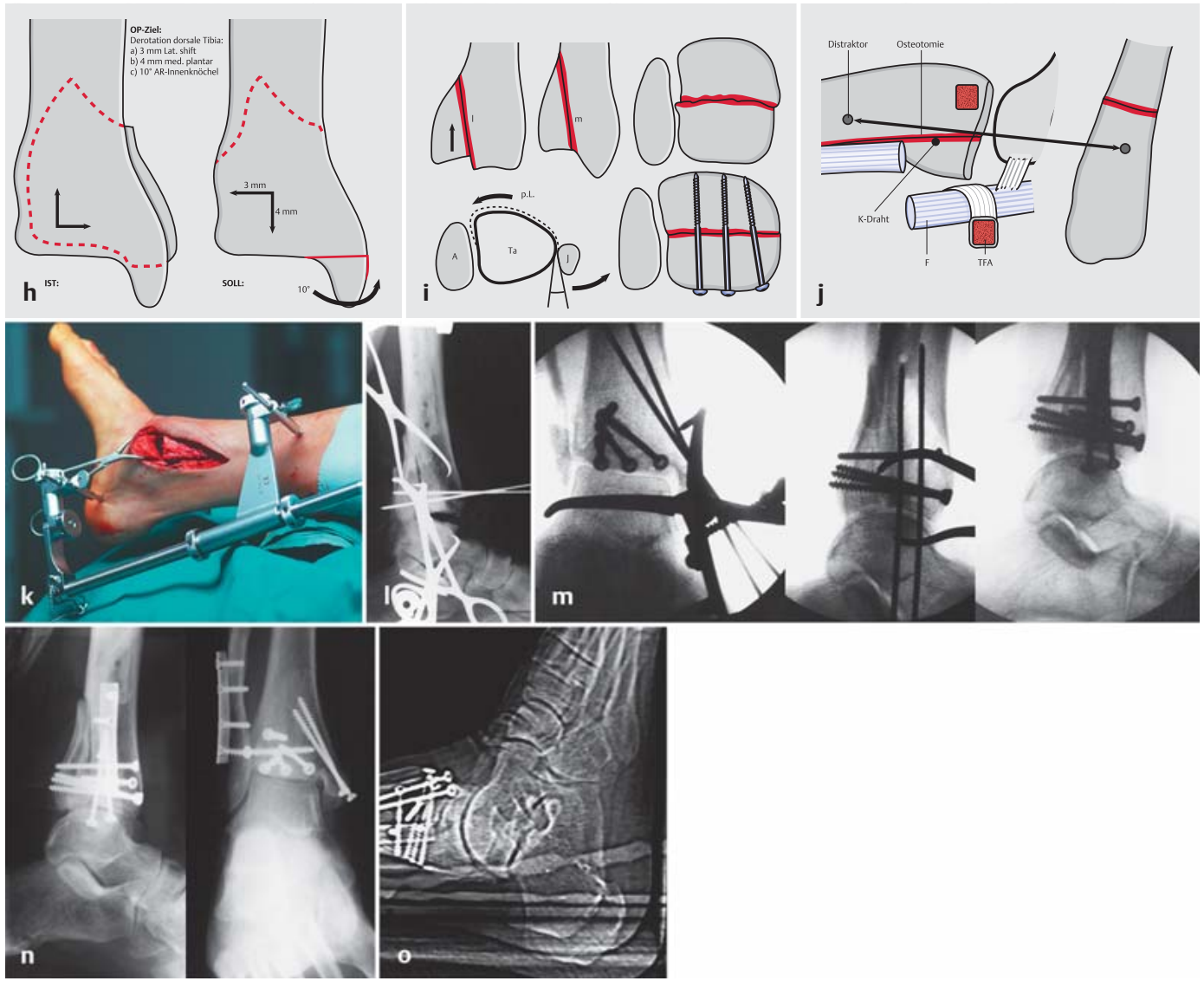

Abb. $\mathbf{8} \mathbf{h}$ bis o Die präoperative Operationsplanung $(\mathbf{h}-\mathbf{j})$ zeigt den Ist- und Soll-Zustand, wobei entsprechend der CT-Daten die dorsale Tibia nach Osteotomie um $3 \mathrm{~mm}$ nach lateral geshiftet und medial um $4 \mathrm{~mm}$ plantarisiert werden soll. Zusätzlich soll durch Innenknöchelosteotomie die $10^{\circ}$ Außenrotationsfehlstellung korrigiert werden. Zur sicheren Osteotomie des fehlverheilten Volkmann ist es notwendig, die Fibula supramalleolar zu osteotomieren. Durch knöchernes Auslösen des Lig. tibiofibulare anterius an der Tibia und Abdrängen der Fibula nach dorsal gelingt die Visualisation des Gelenkspalts, insbesondere durch Einsetzen eines Femurdistraktors. Nur dadurch kann nach Osteotomie die Reposition des großen Volkmann-Fragments zur Wiederherstellung der Gelenkkongruenz ermöglicht werden (k, I). Die intraoperativen Durchleuchtungsaufnahmen (m) zeigen nach temporärem Halten mit Spickdrähten die schrittweise Retention mit 4 Schrauben. Die postoperativen Bilder einschließlich CT (n, o) zeigen die anatomische Rekonstruktion mit zusätzlicher Stabilisierung des Außen- und Innenknöchels.
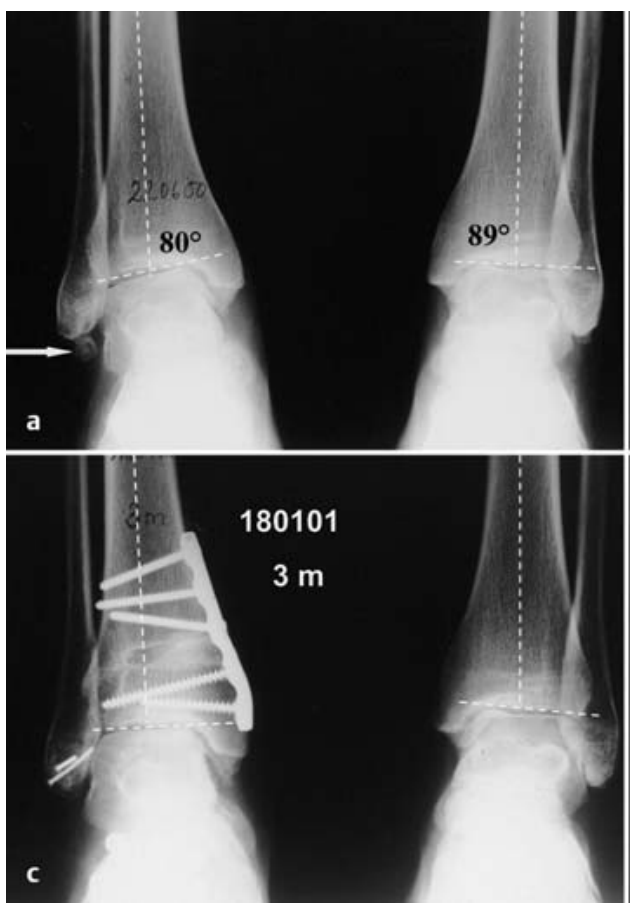

180101

$3 \mathrm{~m}$

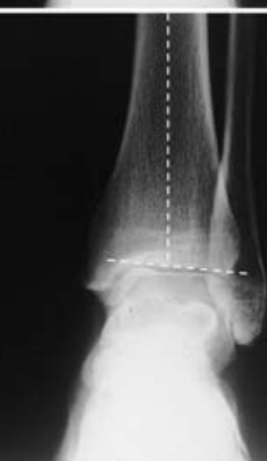

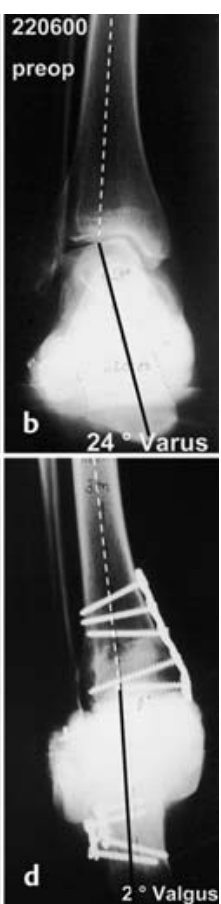

Abb.9a bis d Spezielle Doppelosteotomie supramalleolar (Opening Wedge) nach Dwyer-Osteotomie des Kalkaneus (Closing Wedge) bei OSG- und Rückfußvarus, kombiniert mit chronischer anterolateraler Rotationsinstabilität des oberen Sprunggelenks. Bei einer 25-jährigen Patientin besteht ein alter fibularer ossärer Bandausriss, der als rundlicher Kern subfibular zu erkennen ist (a). Der LDTA beträgt rechts $80^{\circ}$ gegenüber $89^{\circ}$ links. Die Rückfußaufnahmen nach Saltzman (b) zeigen einen insgesamt resultierenden Rückfußvarus von $24^{\circ}$. In solchen seltenen Fällen wird im eigenen Vorgehen eine Doppelosteotomie supramalleolar im Sinne des Opening Wedge $\left(10^{\circ}\right)$ im Anschluss an eine $10-15^{\circ}$ valgisierende Fersenbeinosteotomie nach Dwyer durchgeführt. Der dabei am Kalkaneus gewonnene Knochenkeil $\left(10-15^{\circ}\right)$ wird supramalleolar im Sinne der Opening-Wedge-Korrektur eingesetzt. Neben dieser Maßnahme ist die Rekonstruktion des fibularen Bandapparats essenziell. Im gezeigten Fall wird das große rundliche Fragment, an dem alle 3 Bänder fixiert sind, in eine zu schaffende Grube am Vorderrand des Außenknöchels unter guter Vorspannung in die Grube reponiert und mit 2 Minischrauben retiniert (c, d). Dies führt in der Gesamtheit zu stabilen fibularen Bandverhältnissen und einer korrekten Rückfußachse. Ein rezidivierendes supinatorisches Umknicken wird dadurch sicher vermieden. 

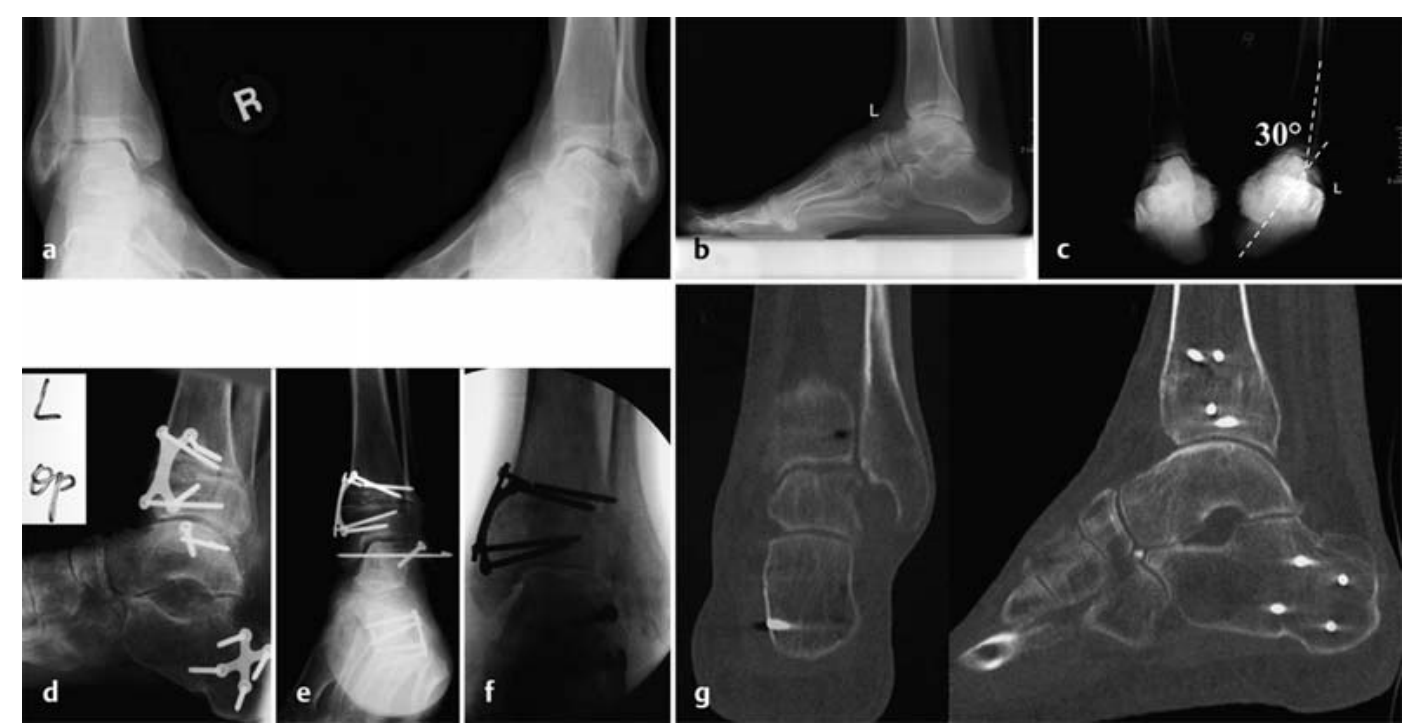

Abb. 10 a bis $\mathbf{g}$ Spezielle Doppelosteotomie bei chronischer anterolateraler Rotationsinstabilität mit fixierter Varuskippung des Talus in der Sprunggelenksgabel. Das Beispiel eines 40-jährigen Patienten mit chronischer Instabilität des fibularen Bandapparats links nach jahrelangem Fußballspiel („footballer’s ankle“) zeigt in der Belastungsaufnahme des oberen Sprunggelenks a.-p. im Stehen die varische Kippung des Talus um $10^{\circ}$ mit erheblicher Fehlbelastung im medialen Kompartiment (a, b). Die Rückfußaufnahme nach Saltzman lässt einen Rückfußvarus von insgesamt $30^{\circ}$ links erkennen. Das operative Prozedere (d-f) ist ähnlich wie im Fallbeispiel 9, d. h. der durch die valgisierende Dwyer-Osteotomie des Fersenbeins gewonnene, keilförmige kortikospongiöse Span von $15^{\circ}$ wird zur supramalleolaren Open-Wedge-Osteotomie $\left(15^{\circ}\right)$ verwandt. Bei der fixierten varischen Fehlstellung des Talus ist es jedoch unerlässlich, das kontrakte Deltoid der oberflächlichen und tiefen Schicht scharf zu lösen, um die varische Kippung korrigieren zu können. Zusätzlich wird mit einem gedoppelten Periostlappen das insuffiziente Lig. fibulotalare anterius ersetzt, welches mit einer Schraube unmittelbar am talaren Ansatz fixiert wird. Um die korrigierte Talusposition bei diesem 2-zeitigen Vorgehen zu halten, muss ein querer K-Draht (2,0 mm Titan) temporär für 6 Wochen vom Innenknöchel transtalar zum Außenknöchel gesetzt werden. Die 1-Jahres-Kontrolle (g) zeigt die anatomische Wiederherstellung des oberen Sprunggelenks und Rückfußes bei noch liegenden Implantaten supramalleolar und im Kalkaneus.

\section{Literatur}

1 Cobey JC. Posterior roentgenogram of the foot. Clin Orthop Relat Res 1976; 118: 202-207

2 Conradi H, Gras U. Ergebnisse nach Korrekturosteotomien posttraumatischer Fehlstellungen der Region des oberen Sprunggelenkes. In: Hierholzer G, Müller KH, Hrsg. Korrekturosteotomien nach Traumen an der unteren Extremität. Berlin, Heidelberg, New York: Springer; 1984: 330

3 Hörster G. Korrekturosteotomien am Tibiaschaft. In: Hierholzer G, Müller KH, Hrsg. Korrekturosteotomien nach Traumen an der unteren Extremität. Berlin, Heidelberg, New York: Springer; 1984: 135

${ }^{4}$ Marti R, Besselaar PP, Raaymakers E. Fehlstellungen nach Verletzungen der distalen Tibiaund Fibulaepiphysen. Orthopäde 1991; 20: 367

5 Miller SD. Late corrections after failed treatment for ankle fractures. Orthop Clin North Am 1995; 26: 363

6 Paley D. Principles of Deformity Correction. Berlin, Heidelberg, New York: Springer; 2002
7 Saltzman CL, El-Khoury GY. The hindfoot alignment view. Foot Ankle Int 1995; 16: 572-576

${ }^{8}$ Takakura $Y$, Tanaka $Y$, Kumai T et al. Low tibial osteotomy for osteoarthritis of the ankle: J Bone Joint Surg [Br] 1995; 77: 50

9 Tarr RR, Resnick CT, Wagner KS et al. Changes in tibiotalar joint contact areas following experimentally induced tibial angular deformities. Clin Orthop Relat Res 1985; 199: 72

10 Verheyden P, Josten C. Supramalleoläre Korrekturosteotomien. Chirurg 1998; 69: 11781187

11 Weber D, Fritschy D, Friederich NF et al. Korrekturosteotomie der distalen Fibula bei posttraumatischer Fehlstellung. Operat Orthop Traumatol 2001; 13: 292-304

12 Weller S. Indikation und Technik der Korrekturosteotomie an der distalen Tibia und der Knöchelgabel. In: Hierholzer G, Müller KH, Hrsg. Korrekturosteotomien nach Traumen an der unteren Extremität. Berlin, Heidelberg, New York: Springer; 1984: 301

13 Yablon IG, Leach RE. Reconstruction of malunited fractures of the lateral malleolus. J Bone Joint Surg [Am] 1989; 71: 521

\section{Prof. Dr. med. Hans Zwipp}

Klinikdirektor

Prof. Dr. med. René Grass,

Ltd. Oberarzt

Prof. Dr. med. Stefan Rammelt

Oberarzt

Klinik und Poliklinik für Unfall-

und Wiederherstellungschirurgie

Universitätsklinikum Dresden

"Carl Gustav Carus“

Fetscherstraße 74

01307 Dresden

uwch@uniklinikum-dresden.de

claudia.vonburski@

uniklinikum-dresden.de 